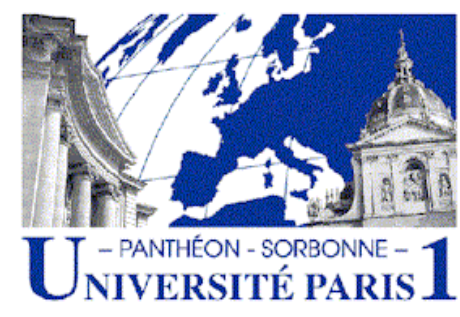

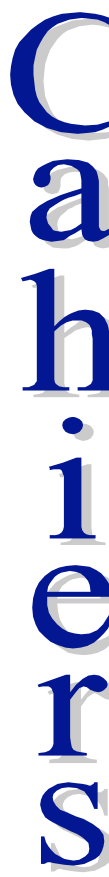

de

la
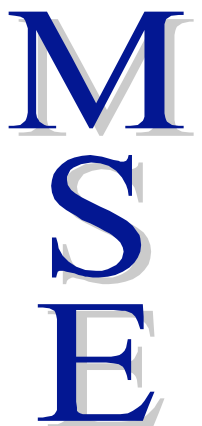

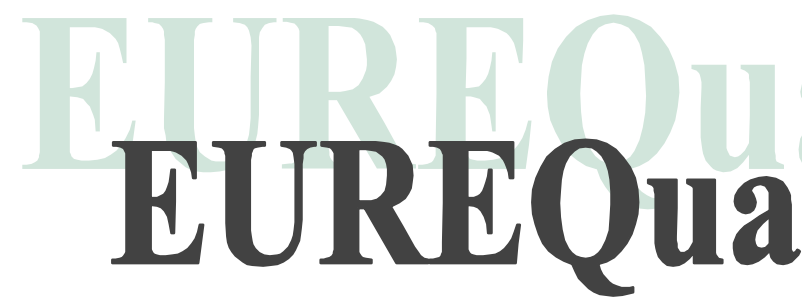

Equipe Universitaire de Recherche en Economie Quantitative - UMR 8594

Une approche comportementale de l'évaluation contingente

Emmanuel FlachaIRE, EUREQua

Guillaume HOLLARD, OEP

2005.77 


\title{
Une approche comportementale de l'évaluation contingente
}

\author{
Emmanuel Flachaire \\ Eurequa, Université Paris 1 Panthéon-Sorbonne \\ et \\ Guillaume Hollard \\ OEP, Université de Marne la Vallée
}

Septembre 2005

\begin{abstract}
Résumé
La théorie économique propose un certains nombre de modèles qui visent à déterminer le montant optimal de bien public à produire sur la base des utilités individuelles. Pour avoir une portée pratique, ces modèles nécessitent de connaître le taux marginal de substitution entre bien privé et bien public. Pour cela, le recours à l'évaluation contingente s'est généralisé à travers le monde. Le problème principal posé par l'évaluation contingente est sa sensibilité à des biais. C'est-à-dire que des paramètres, qui en théorie ne devraient pas influer sur les réponses, ont en pratique une influence. Cet article propose une méthode inspirée de la psychologie sociale qui permet d'identifier les individus les moins sujets aux biais affectant l'évaluation contingente. Deux applications empiriques sont présentées.
\end{abstract}

Ce travail a été rendu possible grâce à l'aide active et efficace de L. Collin et Y. Lichtenberger de l'université de Marne la Vallée. J.M. Tallon, J. Shogren et un rapporteur anonyme ont bien voulu nous faire part de leurs remarques, qu'ils en soient ici remerciés. Les avertissements d'usage s'appliquent. 


\section{Introduction}

La théorie économique propose un certains nombre de modèles qui visent à déterminer le montant optimal de bien public à produire (Stiglitz 2000). Le calcul du montant optimal de bien public est basé sur les utilités individuelles. Pour que ces modèles aient une dimension pratique, il est donc nécessaire d'être capable de mesurer les utilités individuelles. Plus précisément, ce qui intéresse le modélisateur, c'est de connaître le taux marginal de substitution entre bien privé et bien public.

Pour obtenir ces informations, deux grandes catégories de méthodes sont utilisées. Les méthodes indirectes, basées sur des actions observées, et les méthodes directes, basées sur des déclarations individuelles. Les méthodes indirectes consistent à inférer les utilités à partir de comportements observés. Par exemple, un site naturel pour la visite duquel les individus sont prêts à supporter des coûts de transports élevés sera réputé avoir une valeur élevée pour ces individus.

Les méthodes directes consistent, elles, à interroger les agents au moyen de questionnaires. Elles sont notamment mises en oeuvre lorsque les actions observables sont trop confuses, difficilement observables, voire absentes. Par exemple, les côtes d'Alaska, endommagées par le naufrage du pétrolier Exxon Valdez, ont aux yeux des américains une valeur liée à leur existence beaucoup plus qu'à leur usage. C'est pourquoi, pour calculer les dommages réclamés à Exxon par les autorités américaines, la justice eut recours à une évaluation directe, en l'occurrence l'évaluation contingente.

L'évaluation contingente est aujourd'hui largement pratiquée à travers le monde, même si la France fait relativement figure d'exception en la matière. Les applications de cette méthode d'évaluation sont nombreuses, notamment dans les domaines de l'environnement et de la santé. Les pratiques se sont standardisées et répondent à des critères précis et l'usage répété permet de beaucoup mieux cerner les avantages et défauts des méthodes directes. Du succès de la méthode d'évaluation contingente dépend directement la capacité des théories fondées sur les utilités individuelles à proposer des recommandations pratiques en matière de production de bien public.

Le modèle qui sert de soubassement à l'évaluation contingente postule que les individus auraient, préalablement à l'enquête, une valeur parfaitement déterminée enfouie en eux. Or, certains paramètres, qui selon cette théorie ne devraient pas influer sur les réponses, ont en pratique une influence sur les réponses aux enquêtes d'évaluation contingente. Ces violations répétées des prédictions du modèle théorique reçoivent le nom de biais.

Les développements récents de l'économie comportementale (Camerer et al. 2003) proposent de rendre compte des biais affectant la décision. La stratégie de recherche mise en oeuvre consiste à ajouter aux modèles habituels un degré de liberté. Ce surcroît de flexibilité doit permettre de gagner en réalisme et de mieux rendre compte des comportements observés. Idéalement, un simple paramètre supplémentaire suffirait à combler l'écart entre comportements prédits et comportements observés. L'économie comportementale en modélisant le comportement individuel de manière plus réaliste permet de 
corriger à posteriori les informations obtenues de manière à éliminer l'effet des biais. En d'autres termes, il devient possible de reconstituer ce qu'auraient été les choix en l'absence de biais. L'objectif est en quelque sorte d'extraire "la composante rationnelle" des comportements observés. On conçoit dès lors l'attrait que peut exercer une telle approche pour un domaine de recherche dont l'objectif est la révélation des préférences au moyen d'enquête.

Aussi dans cet article, nous proposons une approche comportementale de l'évaluation contingente. Après avoir rappelé les grands principes de l'évaluation contingente et les principaux biais mis en évidence (partie 2), nous proposons une nouvelle approche inspirée de la psychologie sociale, qui vise à extraire davantage d'information afin d'identifier les individus les moins sujets aux biais (partie 3). Nous présentons deux applications centrée sur deux biais particulier : le biais d'ancrage et la divergence wta/wtp.

\section{L'évaluation contingente selon le NOAA panel}

En l'espace de vingt cinq ans, les méthodes d'évaluation contingente sont passées du rang de hobby pour économistes à celui de méthode officiellement reconnue par les tribunaux américains pour évaluer les biens hors marchés. Le chiffre de 10000 évaluations contingentes menées dans le monde paraît vraisemblable. Ce changement de statut doit beaucoup à une modification de la loi américaine en 1980. À cette date, un principe "pollueur payeur" a été inscrit dans loi américaine (Comprehensive Environmental Response, Compensation and Liability Act). En vertu de cette loi, les entreprises peuvent être condamnées pour des dommages infligés à l'environnement. La question se pose alors d'évaluer ces dommages en termes monétaires. Le naufrage de l'Exxon Valdez, qui provoqua une importante marée noire sur les côtes d'Alaska en 1989, fournit un exemple emblématique du changement apporté par cette loi. Vu l'importance des sommes en jeu dans des catastrophes de cette ampleur, il convenait de codifier le plus rigoureusement possible les méthodes d'évaluation. Pour cela, le NOAA (National Oceanic and Atmospheric Administration), chargé de ce dossier, a réuni un panel d'experts présidé par deux prix Nobel, Kenneth Arrow et Robert Solow. Il en a résulté des recommandations précises sur la forme que doit revêtir une bonne évaluation contingente pour être jugée valide par les tribunaux (Arrow et al. 1993). Le rapport final remis par les auteurs prend acte du fait qu'un certain nombre de critiques, parfois virulentes, ont pu être adressées aux méthodes d'évaluation contingente. Ils pensent toutefois qu'il est possible d'élaborer une méthode qui soit raisonnablement à l'abri de ces critiques. C'est ainsi que naît l'évaluation contingente dans sa forme actuelle.

Les recommandations du NOOA se sont imposées comme un point de repère essentiel dans la pratique et l'appréciation de l'évaluation contingente. En focalisant l'attention, le rapport du NOOA a également focalisé les critiques adressées aux méthodes d'évaluation monétaire. Il en a résulté de vigoureux débats (Hausman 1993), qui se prolongent aujourd'hui encore, voire s'amplifient. La controverse est particulièrement intense sur le fait de savoir si les réponses fournies aux questions d'évaluation peuvent raisonnablement 
être prises pour argent comptant, ou si les défauts affectant cette méthode la rendent inopérante.

Dans cette section, nous proposons de rappeler les grands traits qui guident la mise en œuvre de l'évaluation contingente et les difficultés spécifiques qui en découlent, avant de présenter plus particulièrement les biais qui affectent cette méthode directe d'évaluation.

\subsection{L'évaluation contingente en pratique}

L'évaluation contingente se présente sous la forme d'un questionnaire. Préalablement à celui-ci, un scénario décrit en détails la politique ou le bien à évaluer. L'enquêté a toutes les raisons de penser que les autorités publiques envisagent réellement la mise en oeuvre de la politique décrite. L'évaluation est donc contingente au scénario présentant la politique ou le bien en cause. On présente ensuite à l'enquêté un véhicule de paiement, c'est à dire un mécanisme de taxation. Par exemple, la taxe peut être prélevée via la facture d'eau ou d'électricité ou, si le bien s'y prête, en introduisant un péage (pour des parcs naturels par exemple). On pose ensuite aux enquêtés une série de questions relatives à leur pratique en lien avec la politique à évaluer. S'y ajoutent des questions d'identification standard (sexe, age, CSP, ...). Enfin, on en arrive aux questions d'évaluation qui demandent explicitement aux enquêtés jusqu'à quel montant ils accepteraient d'être taxés pour voir la politique décrite mise en oeuvre (ou à quel montant il faudrait les indemniser pour qu'ils renoncent à une politique existante).

Le format des questions posées a fait l'objet d'une attention particulière. Le NOOA panel recommande un format de type referendum. Il s'agit d'une question fermée (seriezvous prêt à payer $x$ euros ?) sous forme d'une proposition chiffrée, à prendre ou à laisser. Ce mode de révélation des préférences possède plusieurs avantages :

- Il permet de ramener l'individu à une situation habituelle dans le domaine de la consommation (acheter ou non à un prix donné) et dans le domaine politique (voter pour ou contre une proposition donnée).

- D'un point de vue cognitif, ce format repose sur une question fermée (on ne peut répondre que "oui" ou "non"), c'est à dire un problème de choix le plus simple possible.

- Enfin, ce mode de révélation est incitatif au sens où il n'offre aucune opportunité de comportement stratégique.

D'autres modes d'élicitation ont été utilisés, notamment les questions ouvertes. Comme nous le verrons par la suite, le choix d'un format particulier, question ouverte ou fermée en particulier, n'est pas sans incidence sur le type de biais susceptible d'affecter les réponses.

Avant d'en venir à l'étude des biais, plusieurs difficultés spécifiques à notre étude doivent être notées :

- L'évaluation contingente consiste en une enquête coûteuse, notamment du fait de la nécessité d'exposer en détails le projet à évaluer. De ce fait, il s'agit d'une 
technique d'enquête qui limite la quantité des données disponibles, notamment par rapport à des expériences de laboratoires menées avec des étudiants. Par exemple, il n'est pas possible de répéter la tâche d'évaluation à plusieurs reprises, ce qui empêche d'étudier l'effet d'un apprentissage.

- Le recours à un questionnaire implique que l'on ne peut obtenir de l'information supplémentaire sur les caractéristiques individuelles qu'en ajoutant une question. Il n'est par exemple pas très réaliste de penser que l'on pourrait comme dans les expériences de laboratoire faire effectuer un certain nombre de tâches aux sujets pour en tirer des informations supplémentaires. Dans notre optique d'économie comportementale, qui consiste à prendre en compte davantage d'information sur les comportements individuels, nous sommes limités par la quantité et la nature de l'information disponible.

- L'information collectée au moyen d'une évaluation contingente est assez fruste. Lorsqu'il s'agit de question fermées, (seriez-vous prêt à payer $x$ euros ?), on obtient une réponse oui/non. Comment attester qu'un simple "oui" ou "non" résulte d'un biais de comportement?

- Enfin, l'évaluation contingente propose un mode de financement particulier pour les biens publics, celui d'une cotisation qui peut être rejeté pour des raisons éthiques. Par exemple, les enquêtés peuvent penser qu'il appartient à d'autres qu'eux de payer pour la politique présentée (par exemple dans le cas de pollution transfrontalière ou lorsque la politique évaluée est traditionnellement financée par l'État). Ces réponses peuvent traduire soit un désintérêt total pour la politique proposée ou, plus vraisemblablement un refus du mode de financement proposé. Le NOAA panel avait réservé une attention particulière aux réponses "zéro" pour prendre en compte les réponses de protestation. Nous montrerons plus loin que des individus ayant des objections peuvent être conduis à diminuer le montant qu'ils sont prêts à payer sans forcément aller jusqu'à refuser complètement de contribuer.

Les critiques les plus fortes adressées aux enquêtes d'évaluation contingente s'appuient sur l'existence de biais. À l'heure actuelle, deux biais retiennent l'attention des chercheurs et donnent lieu aux plus amples débats : le biais d'ancrage et la divergence WTA/WTP. Ce sont notamment ceux qui ont été jugés les plus dramatiques par les opposants à l'évaluation contingente. Aussi, nous les présentons en détails dans les soussections suivantes.

\subsection{Le biais d'ancrage}

L'ancrage est un phénomène général qui apparaît dans les situations d'évaluation de grandeurs objectives (la taille du plus grand séquoia, la hauteur d'une montagne, etc.), ou subjectives (la valeur accordée à un bien dans le cas de l'évaluation contingente). Habituellement, ce biais est mis en évidence dans un dispositif expérimental basé sur deux questions successives. Une première question fermée demande au sujet de comparer son estimation à une valeur fournie par l'expérimentateur, nommée ancre. Une question ouverte demande ensuite au sujet son estimation de la grandeur à évaluer. Il existe une 
forte corrélation entre les ancres fournies et les estimations proposées par les sujets. Tout se passe comme si les sujets ancraient leur réponse à la valeur initialement proposée.

Au plan théorique, une première analyse attribue l'ancrage au fait que les sujets perçoivent l'ancre fournie comme une information sur la valeur de la cible. Un modèle de comportement bayésien conduirait alors le sujet à rapprocher son estimation de la cible. Cette analyse résiste mal à un supplément d'expérimentation. Une série d'expériences utilise des ancres totalement aléatoires, fournies par les sujets eux-mêmes. On demande par exemple aux sujets de comparer leur estimation aux deux derniers chiffres de leur numéro de téléphone. Dans un tel protocole, le sujet peut difficilement supposer que l'ancre contient la moindre information plausible. Pourtant, l'effet d'ancrage persiste. On retrouve un résultat identique en fournissant des ancres non informatives (Mussweiler et Strack 2001). Par exemple, on demande aux sujets s'ils pensent que Gandhi est mort avant l'âge de 140 ans. La quasi totalité des sujets répond par l'affirmative. Or, les estimations fournies ensuite par ces sujets se révèlent nettement plus élevées que celles de sujets à qui l'on a au préalable demandé s'ils pensaient que Gandhi était mort après l'âge de 9 ans. Pourtant, dans les deux cas, l'ancre n'est pas informative (au sens où elle n'apporte aucune information supplémentaire). Plus étonnant encore, lorsque l'on demande après coup aux sujets s'ils pensent avoir été victimes d'un tel effet, une très large majorité est persuadée qu'elle n'a pas été influencée, alors qu'à l'évidence elle l'a été (Ariely et al. 2003). La minorité qui reconnaît avoir été influencée ne parvient pas à corriger ses réponses pour se défaire de l'effet d'ancrage. Des expériences antérieures montrent de même qu'un avertissement préalable ne suffit pas non plus à éviter l'effet d'ancrage (Wilson et al. 1996). De même, l'ancrage persiste lorsque les sujets achètent effectivement le bien avec leur propre argent (Ariely et al. 2003) et même des professionnels avertis (en l'occurrence des experts immobiliers chargés d'estimer un bien) sont sujets au biais d'ancrage (Northcraft et Neale 1987). Le biais d'ancrage est donc un phénomène robuste et difficile à supprimer.

Dans le cadre de l'évaluation contingente, le biais d'ancrage s'applique aux questions d'évaluation qui proposent un montant explicite ("seriez-vous prêt à payer $x$ euros pour la mise en place de cette politique?"). Le montant proposé - le $x$ donc - peut influencer fortement les réponses fournies dans les enquêtes d'évaluation contingente. Changer les $x$ entraîne une variation non négligeable des évaluations recueillies. C'est ce phénomène que l'on qualifie de biais d'ancrage dans les enquêtes d'évaluation contingente. On comprend dès lors que le biais d'ancrage jette un sérieux doute sur les évaluations contingentes qui opèrent aux moyens de questions fermées. Herriges et Shogren (1996) proposent une étude détaillée de l'impact des phénomènes d'ancrage sur les réponses à des questions d'évaluation.

La mise en évidence de ce phénomène n'est pas aussi simple que dans les expériences de laboratoire. En effet, le biais d'ancrage peut être mis en évidence en posant une question fermée ("accepteriez-vous de payez $x$ euros ?"), puis une question ouverte ("combien seriez-vous prêt à payer"). Pour tester la présence d'ancrage, il suffit alors de calculer le coefficient de corrélation entre les montants proposés à la première question et les réponses fournies par les enquêtés à la question ouverte. Mais le format retenu par le 
NOAA panel conduit en effet à poser des questions fermées uniquement. L'inconvénient de ce type de question est qu'un individu qui accepte le principe d'un paiement de 10 euros révèle seulement que son consentement à payer excède ce montant. Une unique question fermée n'amène donc que peu d'information. De plus, la présence d'ancrage ne peut être mise en évidence sur la base d'une unique réponse oui/non. Afin d'améliorer la précision des enquêtes, on utilise alors un format avec deux questions fermées successives. Celui-ci consiste à poser une seconde question en modifiant le montant proposé, à la hausse ou à la baisse, en fonction des réponses apportées (si l'individu accepte de payer un montant donné, on lui propose un montant supérieur; s'il refuse, on lui propose un montant inférieur). On obtient une meilleure précision en situant mieux le consentement à payer.

Herriges et Shogren (1996) montrent que l'ancrage peut être détecté, dans les enquêtes d'évaluation contingente, en étudiant l'influence des montants proposés sur les réponses à la seconde question. Ils proposent un modèle qui permet de corriger le biais d'ancrage, et qui donne une indication de l'ampleur des phénomènes d'ancrage. Leurs résultats empiriques mettent en évidence que, sur certains échantillons l'ancrage semble inexistant, tandis que sur d'autres il semble assez fort, au point que l'on puisse se demander si les réponses obtenues ne sont pas entièrement déterminées par les montants fournis. Dans ce cas, l'ancrage déforme tellement l'information apportée par la seconde question que les auteurs recommandent de renoncer à exploiter celle-ci.

\subsection{La divergence WTA/WTP}

Ce qu'une enquête d'évaluation contingente cherche à déterminer, c'est le montant maximum que les agents accepteraient de payer. C'est à dire la somme au delà de laquelle les agents préfèreraient renoncer au bien ou à la politique proposée. Ce montant maximum reçoit le nom de consentement à payer, ou wtp en référence à l'acronyme de l'expression anglaise "willingness to pay". Le consentement à payer représente une sorte de valeur fondamentale issue des préférences de l'individu. Le but de l'évaluation contingente est donc de rendre accessible cette valeur fondamentale à l'observateur. Pour cela, le plus évident semble de proposer un paiement en échange de la fourniture d'un bien, en mimant ainsi une transaction marchande qui consiste à acheter ce bien. Mais il est également possible de demander à un agent à quelle hauteur il faudrait l'indemniser pour qu'il accepte de renoncer au bien considéré. Le montant minimal à partir duquel les agents renonceraient est nommé wta en référence au terme anglais "willingness to accept". La théorie économique élémentaire suppose que wta et wtp s'égalisent à un niveau où l'individu n'a aucune préférence entre possèder l'objet et possèder la somme correspondante.

Pourtant, il est apparu qu'adopter l'un ou l'autre des modes d'interrogation n'était pas neutre dans le cadre de l'évaluation contingente. Brookshire et Coursey (1987) montrent que les écarts obtenus selon que l'on a recours à l'une ou l'autre méthode peuvent être importants, d'où le terme de divergence entre wta et wtp. Les années récentes ont vu la publication de plusieurs méta-analyses sur la divergence wta/wtp (Plott 
et Zeiler 2005, Sayman et Öncüler 2005). Il s'agit d'analyser simultanément l'ensemble des expériences testant la divergence wta/wtp, aussi bien dans des enquêtes d'évaluation contingente que dans des expériences de laboratoires. Ces données portent de ce fait sur des biens très divers. On recense une cinquantaine d'expériences sur le sujet, chacune incluant différentes versions. Au total, les auteurs ont rassemblé environ 200 données. Le principal enseignement à en tirer est que la divergence est un phénomène général puisqu'elle est présente dans plus de $90 \%$ des expériences analysées. Si l'on mesure l'importance de la divergence en calculant le ratio wta/wtp, on constate que la divergence est souvent de grande ampleur.

L'évaluation contingente tente de connaître le montant qui rend l'individu indifférent entre possèder cette somme et possèder l'objet à évaluer (ou voir la politique à évaluer mise en oeuvre). Or, demander le montant maximum que l'individu serait prêt à payer ou le montant minimal auquel il faudrait l'indemniser ne conduit pas au même résultat. La théorie économique reconnaît essentiellement deux raisons pour lesquelles wta et wtp peuvent diverger. La première concerne l'existence de substituts au bien en cause, la seconde les contraintes de revenu. En l'absence de substituts raisonnables, les agents peuvent en effet exiger un prix minimal (wta) très supérieur à ce qu'ils seraient prêts à payer au maximum pour acquérir ce bien (wtp). Par exemple, Shogren et al. (1994) montrent que, s'agissant de la santé (bien pour lequel il n'y a pas de substitut évident), un agent peut ne pas souhaiter céder un bien qu'il ne pourra pas remplacer. Le wta excède alors largement le wtp. De même, le wtp ne peut excéder le budget d'un individu alors que le wta n'est pas contraint à la hausse. Cependant, même si ces deux effets jouent un rôle important, cela ne suffit pas à expliquer les divergences constatées. La théorie du consommateur standard peine à expliquer l'ensemble des comportements observés.

De ce fait, l'explication la plus souvent avancée pour expliquer les résultats obtenus repose sur la prospect theory (Kahneman et Tversky 1979). Selon ces auteurs, les agents évaluent une situation comparativement à leur situation présente. Leur évaluation d'un objet n'est alors pas indépendante des droits de propriété sur cet objet. Renoncer à un objet que l'on possède est par nature différent d'acquérir ce même objet. Il en résulte que lorsque l'on demande à des individus ce qu'ils seraient prêts à verser pour, en un certain sens, acquérir une politique publique, le résultat serait différent de ce qu'ils seraient prêts à verser pour renoncer à une politique publique dont ils bénéficient déjà.

Un point important est que la divergence est fréquente mais pas systématique. En étudiant en détails les protocoles employés pour mettre en évidence la divergence wta/wtp, Plott et Zeiler (2005) aboutissent à une classification selon quatre conditions : l'anonymat, l'usage d'un mécanisme incitatif pour la révélation des wtp, l'existence de séances d'entraînement et l'usage de paiements réels. La divergence s'atténue lorsque le nombre des conditions satisfaites augmente. Ils mettent alors au point un protocole d'expérience qui permet de satisfaire ces quatre conditions simultanément. L'application de ce protocole expérimental va effectivement annuler la divergence entre wta et wtp dans le cas de bien privé. Or, dans le cas des biens publics qui nous occupent ici (la santé ou l'environnement par exemple), il est difficile d'implémenter une méthode qui suppose une répétition de la tâche à effectuer. 


\section{Une approche comportementale}

L'hypothèse la plus courante postule que les individus sont tous également sensibles à un biais donné. La stratégie de recherche exposée ici remet en cause ce postulat, pour introduire une hétérogénéité individuelle dans la sensibilité aux biais. Avant d'en venir aux multiples problèmes techniques que cela soulève, examinons l'intérêt d'une telle stratégie de recherche.

Comme nous l'avons mentionné dans la section précédente, l'évaluation contingente ne permet pas de laisser aux agents un temps d'apprentissage significatif. Il est donc possible que les préférences révélées soient peu stables dans le temps et excessivement sensibles aux effets de contexte. Supposons, pour simplifier, que l'on puisse identifier un groupe parfaitement insensible aux biais divers affectant l'évaluation contingente. Les réponses fournies par ces individus contiendraient une information substantielle. A l'opposé, les réponses des individus les plus sensibles aux biais ne contiennent que peu d'information intéressante puisque leurs réponses seraient essentiellement conditionnées par l'enquête elle-même. En simplifiant, on obtient une catégorie d'individus dont les réponses sont réellement porteuses d'information sur les préférences individuelles, et une autre dont les réponses contiennent essentiellement un bruit généré par l'enquête. Ne pas distinguer ces deux catégories conduit donc à une perte d'information très sensible.

Au plan technique, cela pose plusieurs problèmes. Comment trouver une proxy pour la sensibilité aux biais, en étant limité dans la collecte d'information à une enquête par questionnaire? Quelle garantie a-t-on que l'information ainsi collectée n'est pas redondante avec les variables usuelles? Enfin, l'hétérogénéité introduite implique d'adapter les modèles économétriques utilisés.

Pour avancer dans la direction envisagée, nous utiliserons une approche inspirée des théories des représentations sociales. Ces théories ont été développées dans le cadre de la psychologie sociale. Au niveau le plus général, la représentation est ce par quoi un objet est présent à l'esprit. Dans le cadre d'une enquête, les représentations sont appréhendées au moyen de questions d'évocation du type "Qu'évoque pour vous tel objet ?". L'analyse structurelle montre que les représentations sociales s'organisent selon une structure particulière (Farr 1998, Vergès 1994). Cette structure se compose d'un noyau central qui contient les lieux communs, les stéréotypes, les éléments habituellement véhiculés dans les média et plus généralement ce que les sociologues nomment le sens commun. Les individus qui utilisent abondamment les éléments contenus dans le noyau central sont qualifiés de conformistes, tandis que ceux qui s'en distinguent sont qualifiés de non conformistes. Cette dernière appellation se justifie du fait que seule une petite minorité parvient à décrire un objet sans avoir recours aux éléments du noyau central, qui sont ceux qui viennent le plus spontanément à l'esprit. Résister à l'attraction du noyau central implique d'avoir une opinion forte et une bonne appréhension de ce que sont les idées reçues sur une question donnée. Cette position de non conformiste peut provenir d'une pratique antérieure (on n'a pas la même représentation du bruit si l'on est riverain d'une autoroute ou d'une voie de chemin de fer) et/ou d'une réflexion plus poussée (par exemple un militant écologiste se distingue en général dans les réponses 
relatives aux questions d'environnement).

Si l'on admet que la préférence d'un individu évolue dans le temps, il est alors possible de considérer sa représentation comme un marqueur des apprentissages passés. Les nonconformistes se caractérisent par le fait qu'ils ont une expérience plus directe de l'objet étudié et/ou une meilleure connaissance de celui-ci. En forçant le trait, on peut imaginer que le processus d'apprentissage a déjà convergé. Les préférences de ces individus seront donc relativement stables. Cette notion compléterait avantageusement la notion d'utilité, qui ne représente les goûts d'un individu que de manière statique à un point du temps.

L'idée que nous exploitons dans ce qui suit est que les non conformistes sont des individus qui ont eu à se construire en opposition à la majorité, et qu'ils sont de ce fait peu influençables. Notre hypothèse centrale est alors que ces individus ont une moindre sensibilité aux effets de contexte dans le cadre d'une évaluation. Notons que le travail d'identification des non-conformistes ne se justifie que dans la mesure où la distinction conformiste/non conformiste ne peut être déduite des variables usuelles (sexe, age, profession, etc.).

\subsection{Le biais d'ancrage}

En évaluation contingente, l'ensemble des modèles proposés pour corriger le biais d'ancrage suppose que ce phénomène affecte de la même manière la population dans son ensemble (Herriges et Shogren 1996, Alberini, Kanninen, et Carson 1997, Whitehead 2002, DeShazo 2002, Flachaire et Hollard 2006). À notre connaissance, aucune recherche ne montre que l'ancrage affecte seulement une partie des individus. Cela provient certainement du fait que les variables usuelles ne font pas apparaître un tel phénomène. La mise en évidence d'une telle hétérogénéité ne peut donc se faire qu'en ayant recours à une variable qui apporte une information différente de celle fournie par les variables usuelles. En faisant appel à la théorie des représentations sociales, nous avons mené des travaux qui mettent en évidence une telle hétérogénéité dans les enquêtes d'évaluation contingente (Flachaire, Hollard, et Luchini 2003 et Flachaire et Hollard 2005). Les modèles que nous avons proposés permettent alors de corriger le biais d'ancrage et d'améliorer de manière significative la précision des estimations.

Dans ces travaux, nous utilisons les données d'une enquête d'évaluation contingente effectuée auprès de 218 visiteurs de la réserve naturelle de la Camargue en 1997. L'enquête consiste à évaluer le montant que seraient prêts à payer les individus pour préserver le site naturel. Le taux de réponses est élevé (92.6\%). Pour une description détaillée des données et de l'enquête, voir Claeys-Mekdade, Geniaux, et Luchini (1999). L'estimation d'un modèle standard et une analyse spécifique permet de mettre en évidence la présence d'un biais d'ancrage. L'utilisation d'un modèle adapté à ce problème, celui développé par Herriges et Shogren (1996), montre que le biais d'ancrage peut être corrigé, mais qu'il s'accompagne d'une perte de précision importante dans l'estimation du modèle. Pour retrouver une estimation plus efficace, nous proposons de prendre en compte une hétérogénéité des individus dans leur comportement face à l'ancrage. Une question d'évocation 


\begin{tabular}{|c|c|c|c|c|}
\hline Variables & \multicolumn{2}{|c|}{ Ancrage standard } & \multicolumn{2}{|c|}{ Ancrage hétérogène } \\
\hline Constante & 83.57 & (68.43) & 61.16 & $(44.18)$ \\
\hline Distance maison-site & 7.07 & $(4.45)$ & 4.67 & $(2.17)$ \\
\hline Visite en voiture & -79.47 & $(49.04)$ & $\star \quad-58.22$ & $(26.81)$ \\
\hline Employé & 84.27 & $(49.09)$ & $\star \quad 65.36$ & $(27.77)$ \\
\hline Classe moyenne & 99.89 & $(56.95)$ & $\star \quad 74.66$ & $(28.96)$ \\
\hline Inactif & 57.12 & $(40.87)$ & 48.80 & $(27.99)$ \\
\hline Ouvrier & 81.27 & $(81.66)$ & 62.00 & $(53.27)$ \\
\hline Cadre & 78.88 & $(44.24)$ & 59.66 & $(24.65)$ \\
\hline Visite en famille & 12.79 & $(31.36)$ & 13.01 & $(22.71)$ \\
\hline Visite seul & 122.37 & (95.03) & 89.18 & $(52.97)$ \\
\hline Visite en groupe & 3.70 & (46.24) & 4.22 & $(32.65)$ \\
\hline Première visite & 18.56 & $(23.50)$ & 15.59 & $(16.31)$ \\
\hline Autres facilités proposés & 57.29 & (33.06) & 41.94 & $(15.59)$ \\
\hline Autres financements proposés & -28.19 & (21.84) & -19.01 & $(12.87)$ \\
\hline Sud-Ouest & -42.04 & $(40.61)$ & -28.48 & $(24.24)$ \\
\hline Sud-Est & 52.72 & $(52.06)$ & 40.73 & $(32.61)$ \\
\hline Type de questionnaire & -13.15 & $(17.82)$ & -10.50 & $(11.97)$ \\
\hline Enquêteur 1 & 6.12 & $(47.50)$ & 8.26 & $(32.07)$ \\
\hline Enquêteur 2 & -39.70 & $(54.49)$ & -29.92 & $(35.09)$ \\
\hline$\gamma:$ paramètre d'ancrage & 0.52 & $(0.22)$ & 0.36 & $(0.14)$ \\
\hline
\end{tabular}

TAB. 1 - Enquête sur la Camargue

est posée à la fin de chaque questionnaire : "Qu'évoque pour vous la Camargue ?". Sur la base de cette question, une méthode de traitement des données permet de classifier les individus en deux groupes, conformistes/non-conformistes. La spécification d'un modèle économétrique adapté, tenant compte d'une sensibilité différente à l'ancrage des deux groupes, donne lieu à un nouveau modèle.

Le tableau 1 présente les résultats économétriques de l'exploitation des données de la Camargue. L'estimation d'un modèle probit à double offre tenant compte d'un ancrage standard (Herriges et Shogren 1996) est présenté dans une première colonne, appelée Ancrage standard. L'estimation d'un modèle probit à double offre tenant compte d'un ancrage hétérogène (Flachaire et Hollard 2005) est présenté dans une deuxième colonne, appelée Ancrage hétérogène. Les résultats de l'estimation montrent que les écart-types du modèle Ancrage hétérogène sont beaucoup plus petits que ceux du modèle Ancrage standard, de telle sorte que sept paramètres estimés sont significatifs dans le modèle hétérogène, contre un seul dans le modèle standard. Ces résultats montrent clairement que la prise en compte d'une sensibilité différente à l'ancrage entre deux groupes distincts permet d'améliorer significativement la précision des estimateurs. 


\subsection{La divergence WTA/WTP}

Pour étudier la divergence wta/wtp dans le cas de biens publics, il est nécessaire de trouver un bien public qui puisse être à la fois acquis et au contraire cédé. Notre choix s'est porté sur un bien particulier, les cours le samedi matin. Au moment de l'enquête, une discussion était engagée à l'université de Marne la Vallée pour étudier des remèdes au manque chronique de salle de cours. Deux solutions étaient envisagées. L'une des solutions consistait à systématiser les cours le samedi matin. La solution alternative consistait à louer des salles à proximité de l'université. De ce fait, nous pouvions présenter de manière crédible aux étudiants un choix entre avoir cours le samedi matin ou contribuer, via une hausse des droits d'inscription, à la location de salles pour éviter les cours le samedi matin. De cette manière, il est possible d'évaluer les consentements à payer (wtp) pour garder libre le samedi matin. Pour évaluer les consentements à recevoir (wta), on s'appuie sur le fait que les locaux de l'université sont couramment utilisés pour des tournages de cinéma. On propose alors de baisser les droits d'inscription grâce à une systématisation de ces tournages. En contrepartie, les cours le samedi matin seront généralisés. On demande alors aux étudiants quelle indemnité compenserait le désagrément d'avoir cours le samedi matin.

L'enquête a été effectuée auprès de 359 étudiants de l'université de Marne la Vallée; 184 étudiants ont répondu à la version wtp et 175 à la version wta. En préambule, il est expliqué que l'université, en réponse à des sollicitations de la part des étudiants, met en place une procédure de décision nouvelle, basée sur les résultats de l'enquête. Il est également précisé que les décisions ne seront prises que si une large majorité d'étudiants y est favorable. L'enquête débute par la lecture d'un texte du président de l'université qui explique la démarche et invite les étudiants à répondre sérieusement. Suivent une vingtaine de questions. La première dizaine porte sur l'évaluation contingente elle-même, la seconde comporte des questions d'identification classiques. Les interviews de sortie indiquent que les étudiants ont répondu en ayant clairement en tête les implications de leurs réponses.

Si on considère l'ensemble des réponses, pour les deux versions du questionnaire, on obtient une moyenne des consentements à payer (wtp) égale à 15.3 euros et une moyenne des consentements à recevoir (wta) égale à 68.7 euros. Cela correspond à un ratio wta/wtp égal à 4.5, une divergence dont l'ampleur est conforme aux résultats habituels (Sayman et Öncüler 2005).

Pour contrôler la divergence dans le cas de biens publics, nous reprenons notre méthode inspirée des représentations sociales, avec comme objectif d'identifier des individus qui n'y seraient pas sensibles. L'inconvénient de la méthode employée dans l'enquête sur la Camargue est qu'elle repose sur une question d'évocation ouverte. Cela impose de traiter une liste de mots. Une des étapes du traitement comporte donc une classification qui peut être jugée ad-hoc. Préalablement à notre enquête, nous avons donc choisi de mener une enquête similaire en question ouverte. Nous sommes partis d'une question d'évocation sur ce que représentaient les études aux yeux des étudiants. Nous avons ensuite isolé un type de réponses qui identifiait un groupe de non-conformiste (au sens où nous 
l'avions defini dans l'enquête précédente). Il est apparu que le clivage, conformiste/nonconformiste, pouvait être relié à une opposition plus générale concernant la finalité des études à l'université. Nous avons alors synthétisé ce travail préalable en une unique question fermée : "Selon-vous, vos études servent prioritairement à : (1) acquérir des compétences; (2) obtenir des diplômes". L'élaboration de cette question d'évocation constitue un résultat en soi sur la représentation que les étudiants se font de l'université. Mais c'est surtout dans le traitement de l'information que l'apport est le plus substantiel. L'ajout de cette question fermée au questionnaire nous permet de classer directement les individus en deux sous-groupes, sans avoir recours à une méthode de classification spécifique.

Nous utilisons également une seconde question plus classique concernant l'appréciation portée sur la procédure elle-même. Le principe de financer par une hausse de droits d'inscription les locaux nécessaires pour éviter les cours le samedi matin peut en effet paraître choquant (cas de la version wtp). De même, louer des batiments universitaires pour des tournages de cinéma peut être jugé innoportun (cas de la version wta). Pour tenir compte de ces éventuelles réserves, nous interrogeons directement les enquêtés sur leur avis concernant la démarche elle-même, qu'ils peuvent au choix juger acceptable, discutable ou inacceptable. Un des résultats les mieux établis dans l'évaluation contingente est que des individus peuvent refuser de contribuer, ou demander des sommes astronomiques, pour manifester ainsi leur opposition à la démarche elle-même. Il a souvent été soupconné, mais jamais testé à notre connaissance, que les individus les plus opposés à la démarche cotiseraient faiblement (et non pas simplement refuseraient complétement de payer). Symmétriquement, dans la version wta les individus les plus opposés demanderaient des montants élevés mais néanmoins raisonnables. De ce fait, la divergence wta/wtp serait d'autant plus forte que les individus ont des réserves à l'égard de la procédure.

Le tableau 2 présente les moyennes des consentements à payer (wtp) et des consentements à recevoir (wta), ainsi que le ratio wta/wtp, ventilés en fonction des réponses aux deux questions, d'évocation et d'avis sur la procédure.

\begin{tabular}{|c|c|c|c|c|c|c|c|}
\hline & \multicolumn{3}{|c|}{ Compétences } & \multicolumn{3}{|c|}{ Diplômes } & \multirow[t]{2}{*}{ Tous } \\
\hline & Accep. & Discu. & Inacc. & Ассер. & Discu. & Inacc. & \\
\hline Moyenne WTP & 56.1 & 17.9 & 6.7 & 20.6 & $\bar{~} 13.3$ & 4.4 & 15.3 \\
\hline Moyenne WTA & 56.2 & 66.8 & 69.4 & 67.7 & 64.0 & 113.6 & 68.7 \\
\hline Ratio WTA/WTP & 1.0 & 3.7 & 10.3 & 3.3 & 4.8 & 25.9 & 4.5 \\
\hline
\end{tabular}

TAB. 2 - Enquête à l'université de Marne-la-Vallée

Les résultats sont explicites et dépassent même nos espérances. Les individus qui jugent acceptable la procédure (Accep.) et qui appartiennent au groupe dont on suspecte qu'il a un avis plus tranché sur la finalité des études (Compétences) n'est pas sensible à la divergence wta/wtp. Dans ce cas, la moyenne des consentements à payer est égale à 56.1 et la moyenne des consentements à recevoir est égale à 56.2. Les six catégories d'individus 
distinguées ici se comportent de manière radicalement différentes : la divergence disparaît pour un groupe (le ratio est égal à 1) alors qu'elle est très importante pour un autre groupe (le ratio atteint 25,9 ).

Les variables construites sur la base de l'analyse qui précède permettent donc de séparer les populations de manière à prévoir leur sensibilité à la divergence wta/wtp. Nos résultats confirment l'intuition selon laquelle il existe une forte hétérogénéité relative à la sensibilité aux biais. Au plan de l'évaluation des biens publics, ce résultat est important. Si on ne tient pas compte d'une telle hétérogénéité, la valeur estimée du bien se situe dans un intervalle de grande amplitude, borné en bas par la moyenne des wtp et en haut par la moyenne des wta : [15.3;68.7] dans notre exemple. Par contre, si on tient compte de l'hétérogénéité et si on se concentre sur le groupe qui n'est pas sensible au biais, l'intervalle obtenu $[56.1 ; 56.2]$ fournit une estimation beaucoup plus fiable et plus précise de la valeur du bien public.

\section{Conclusion}

Pour faire face aux défauts de rationalité, l'économie comportementale propose une stratégie en deux étapes. Dans un premier temps, il convient d'établir des modèles de décision qui montrent comment les biais viennent affecter la décision individuelle. Cela permet ensuite, de "débiaiser" les comportements observés ex-post. En d'autres termes, on extrait la composante rationnelle des comportements observés. C'est cette approche que nous suivons ici. Notre apport porte principalement sur un raffinement de l'approche comportementale qui consiste à distinguer les individus en fonction de leur sensibilité aux biais. Cela permet de beaucoup mieux cibler le "débiaisage". Nos résultats empiriques se traduisent par une meilleure estimation des modèles économétriques d'évaluation contingente.

\section{Références}

Alberini, A., B. Kanninen, et R.T. Carson (1997). Modeling response incentive effects in dichotomous choice valuation data. Land Economics, 73, 309-324.

Ariely, D., G. Loewenstein, et D. Prelec (2003). "Coherent arbitrariness : Stable demand curves without stable preferences". Quarterly Journal of Economics, 118-1, 73-105.

Arrow, K., R. Solow, P. R. Portney, E. E. Leamer, R. Radner, et H. Schuman (1993, January). Report of the NOAA panel on contingent valuation. Technical Report, 58, p1601-1614.

Brookshire, D. et D. Coursey (1987). "Measuring the value of a public good : an empirical comparaison of elicitation procedures. American Economic Review, 77, 554-556.

Camerer, C., G. Loewenstein, et M. Rabin (2003). Advances in Behavioral Economics. Princeton Press. 
Claeys-Mekdade, C., G. Geniaux, et S. Luchini (1999). Quelle valeur attribuer à la Camargue? Une perspective interdisciplinaire économie et sociologie. In P. Point (Ed.), La valeur économique des hydrosystèmes. Méthodes et modèles d'évaluation des services délivrés, Chapter 7, pp. 137-168. Paris : Economica.

DeShazo, J. R. (2002). "Designing transactions without framing effects in iterative question formats". Journal of Environmental Economics and Management, 43, p360-385.

Farr, R. M. (1998). From collective to social representations : Aller et Retour. Culture and Psychology, 4, 275-296.

Flachaire, E. et G. Hollard (2005). "Individual sensibility to framing effects in surveys". mimeo.

Flachaire, E. et G. Hollard (2006). "Controlling starting-point bias in double-bounded contingent valuation surveys". Land Economics, 81, forthcoming.

Flachaire, E., G. Hollard, et S. Luchini (2003). "A new approach to anchoring : theory and empirical evidence from a contingent valuation survey". Cahier de la MSE 2003.109, Université Panthéon-Sorbonne, Paris.

Hausman, J.A. (1993). Contingent valuation : A critical assessment. North-Holland.

Herriges, J. A. et J. F. Shogren (1996). "Starting point bias in dichotomous choice valuation with follow-up questioning". Journal of Environmental Economics and Management, 30, 112-131.

Kahneman, D. et A. Tversky (1979). "Prospect theory : an analysis of decisions under risk". Econometrica, 47, 313-327.

Mussweiler, T. et F. Strack (2001). "Considering the impossible : Explaining the effects of implausible anchors.". Social Cognition, 19, 145-160.

Northcraft, G. et M. Neale (1987). "Experts, amateurs and real estate : an anchoring and adjustement perspective on property pricing decisions". Organizational Behavior and Human Decision Processes, 39, 84-97.

Plott, C. R. et K. Zeiler (2005). "The willingness to pay/willingness to accept gap, the endowment effect, subject misconceptions and experimental procedures for eliciting valuations". American Economic Review, 95, -.

Sayman, S. et A. Öncüler (2005). "Effects of study design characteristics on the wtawtp disparity : a meta analytical framework". Journal of Economic Psychology, 26, $289-312$.

Shogren, J., S. Shin, J. Kliebenstein, et D. Hayes (1994). "Resolving differences in willingness to pay and willingness to accept". American Economic Review, 84, 255-270.

Stiglitz, J. E. (2000). Economics of the Public Sector. W. W. Norton \& Company.

Vergès, P. (1994). Approche du noyau central : propriétés quantitatives et structurales. In Structures et transformations des représentations sociales, Textes de base en Sciences Sociales, Chapter 8, pp. 233-253. Lausanne : Delachaux et Niestlé.

Whitehead, J. C. (2002). "Incentive incompatibility and starting-point bias in iterative valuation questions". Land Economics, 78, p285-297.

Wilson, T. D., C. Houston, K. M. Etling, et N. Brekke (1996). "A new look at anchoring effects : Basic anchoring and its antecedents". Journal of Experimental Psycho$\log y, 4,387-402$. 